epidermal growth factor receptor 2 (HER2) overexpression and programmed death-ligand 1 (PD-L1) positive immunohistochemistry in vulvar Paget disease in Thai women. Methods: A cross-sectional study was conducted in King Chulalongkorn Memorial Hospital, Bangkok, Thailand. Histologically diagnosed patients with vulvar Paget disease from January 2000 to April 2020 were identified. Clinical data were collected from medical records and histologic data were verified by pathological review. Pathologic specimens were collected. HER 2 and PD-L1 immunohistochemistry were performed. The results of staining were reported by two pathologists. Data were analyzed using descriptive statistics and reported as percentage and $95 \%$ confidence interval (CI). Clinical outcomes were correlated with immunohistochemistry expression.

Results: Thirty-three vulvar Paget disease cases were included in the study. Twenty patients $(60.6 \%, 95 \% \mathrm{CI}=42.1-77.1)$ had HER2 overexpression. All patients were negative for PD-L1 immunohistochemistry. HER2 positive patients had hazard of death 2.94 (95\% CI=0.61-14.23) times compared with HER2 negative patients. HER2 positive status had relative risk 1.95 and 1.73 for invasive and metastatic disease respectively.

Conclusion: More than half of vulvar Paget disease patients had HER 2 overexpression and might benefit from anti-HER2 targeted therapy. A higher proportion of HER2 overexpression was found in invasive and metastatic vulvar Paget disease. PD-L1 was not expressed in vulvar Paget disease.

Poster (CO4)

Preinvasive Disease of Cervix, Vulva, and Vagina

https://doi.org/10.3802/jgo.2021.32.S1.C04

\section{Is human papillomavirus genotype important in predicting disease progression in women with biopsy-proven negative or CIN1 of LSIL cytology?}

\section{Woodae Kang, * UChul Ju, SeokMo Kim \\ Chonnam National University Medical School, Gwangju, Korea (woodai75@naver.com)}

Objective: Our aim was to estimate the risk of disease incidence in women with low-grade squamous intraepithelial lesion (LSIL) without histology-proven cervical intraepithelial neoplasia grade 2 or worse (CIN2+) by human papillomavirus (HPV) genotype. Methods: Between January 2002 and December 2010, incidence of CIN2+ in 1,580 women including 575 with LSIL and histologyproven negative and 1,005 with LSIL with histology-proven CIN1 was investigated. Baseline high-risk-HPV status was determined by the hybrid capture II assay and high-risk-HPV genotype by the HPV DNA chip test. Cumulative incidence and hazard ratios (HRs) were estimated to explore differences between index data and associations with CIN2+. Results: Of the 1,580 women, 139 (8.8\%) patients developed CIN2+. The 5-year cumulative incidence rate of CIN2+ in HPV-16, HPV-18, and HPV-58 were $18.7 \%, 16.1 \%$, and $12.9 \%$, respectively. On multivariate analysis, being positive in HPV-16 $(\mathrm{HR}=2.324 ; \mathrm{p}<0.01), \mathrm{HPV}-18$ (HR=2.315; $\mathrm{p}<0.01)$, and HPV58 ( $\mathrm{HR}=2.051 ; \mathrm{p}<0.01)$ were significantly associated with developing CIN2+ compared to being negative for that type. Conclusion: Among women with LSIL, HPV-16, HPV-18, or HPV58 positive women may need intensified follow-up as they have the highest risk of becoming CIN2+.

Poster (C05)

Gynecologic Cancer Screening

https://doi.org/10.3802/jgo.2021.32.S1.C05

\section{Liquid-based cytology for cervical cancer screening compared between spatula-cotton swab and spatula-cytobrush: a randomized controlled trial}

\section{Perapong Inthasorn," Wiphawee Hochaame, Somsak Laiwejpithaya, Chatchai Areeswate \\ Siriraj Hospital, Bangkok, Thailand (perapong_i@hotmail.com)}

Objective: The presence of endocervical cell/transformation zone $(\mathrm{EC} / \mathrm{TZ})$ component is still described as a quality indicator in the 2014 Bethesda System. In addition, the presence or absence of $\mathrm{EC} / \mathrm{TZ}$ component is a useful quality assurance measurement. The aim of this study was to compare the percentage of satisfactory samples with presence of EC/TZ components between samples collected by spatula-cotton swab and by spatula-cytobrush for cervical cancer screening using Siriraj liquid-based cytology.

Methods: This randomized controlled trial was conducted during January 2020-March 2020. A total of 1,400 women were recruited. Study women were randomly allocated to either the spatula-cotton swab group or the spatula-cytobrush group (700 women per group). All samples were sent for cytologic interpretation and determination of satisfactory sample with presence of EC/TZ component.

Results: There were no significant differences in baseline characteristics between groups. The percentage of satisfactory samples with presence of EC/TZ components was significantly higher in the spatula-cytobrush group than in the spatulacotton swab group $(87.1 \%$ vs. $75.9 \%$, respectively, $\mathrm{p}<0.001)$. There were no significant differences between 2 groups relative 
to the percentage of satisfactory smears or the rate of detection of abnormal cells.

Conclusion: Our results support the efficacy of spatulacytobrush for cervical cancer screening.

Poster (C06)

Cervical Cancer

https://doi.org/10.3802/jgo.2021.32.S1.c06

\section{Utility of radiomics for predicting patient local control in cervical cancer with chemoradiotherapy}

\section{Wei Jiang, ${ }^{1}$ Haowen Pang, ${ }^{2, *}$ Han Chen ${ }^{2}$}

'Department of Radiotherapy, Yantai Yuhuangding Hospital, Yantai, China ${ }^{2}$ Department of Oncology, The Affiliated Hospital of Southwest Medical University, Luzhou, China (8680003@qq.com)

Objective: The aim of this study was to develop a predictive model combining radiomic features with clinical characteristics for predicting local control (LC) in cervical cancer patients receiving intensity modulated radiotherapy (IMRT).

Methods: This was a retrospective analysis of 161 patients with cervical cancer treated with IMRT, using data acquired between May 2012 and March 2017. We extracted the radiomic features from computed tomography (CT) scans. Least absolute shrinkage and selection operator regression was used to filter the extracted radiomic features and reduce the dimensionality of the data. Rad-score was calculated by the selected radiomic features. Multivariate Cox-regression hazard models was established to analyze the LC of cervical cancer patients, and a Nomogram prediction model based on Rad-score and clinical characteristics was used to demonstrate the prediction model. The prediction reliability was evaluated by the area under the receiver operating characteristic curve and Harrell's concordance index. Decision curve analysis evaluates the application value of the Nomogram. The heat map shows the relationship between radiomics features and clinical characteristics. According to the cut-off value of Radscore, all patients were classified as low-risk or high-risk.

Results: A total of 5 radiomic features and 2 clinical characteristics were extracted for analysis. A combination of the Rad-score and clinical characteristics resulted in better performance for the estimation of 5-year LC (area under the curve $[A U C]=0.780$; confidence interval $[\mathrm{CI}]=0.690-0.870)$ than that with clinical characteristics alone (AUC $=0.680 ; \mathrm{CI}=0.590-0.770$ ). These patients were divided into high-risk and low-risk groups according to the cut-off value of Rad-score.

Conclusion: This study shows that the combination of CT extracted radiomic features and clinical characteristics has a good potential for evaluating LC in patients with cervical cancer treated with IMRT.
Poster (C07)

Cervical Cancer

https://doi.org/10.3802/jgo.2021.32.S1.C07

\section{Pretreatment lymphocyte counts as independent prognostic factors in patients with locally advanced cervical cancer treated with concurrent chemoradiotherapy}

Ekasak Thiangphak, Korrawit Pruegsanusak, Rakchai Buhachat ${ }^{*}$

Songklanagarind Hospital, Hat Yai, Thailand (brakchai@medicine.psu.ac.th)

Objective: To evaluate the association between pretreatment total lymphocyte counts (TLC) and survival in locally advanced cervical cancer patients treated with concurrent chemoradiation (CCRT). Methods: A Retrospective analysis of 789 patients diagnosed cervical cancer stage IIB to IVA who treated with primary CCRT from January 2011 to December 2015 was performed. Pretreatment TLC was evaluated for an association with 5-year disease free survival (DFS) and overall survival (OS) rates. Results: Seven hundred and eighty-nine patients were included in the study. There were 52 patients had pretreatment TLC $<1,000$ cells $/ \mathrm{mm} 3$. The median pre-treatment TLC in groups TLC $<1,000$ cells $/ \mathrm{mm} 3$ and $>1,000$ cells/mm3 were 573.9 cells/ mm3 (range $=350.9-827.7$ cells $/ \mathrm{mm} 3$ ) and 2,211.3 cells/mm3 (range $=1,751.3-2,785.8$ cells/mm3) respectively. Patients in pretreatment TLC $<1,000$ cells/mm3 group had fewer number of treatment response and trend to present in more advance stages of disease and larger tumor size. The 5-year DFS and OS rates were significantly higher in patients with pretreatment TLC $>1,000$ cells/ $\mathrm{mm} 3$ than the counterparts $(67.7 \%$ vs. $35.4 \%$ [ $\mathrm{p}<0.0001], 57.6 \%$ vs. $25.7 \%[\mathrm{p}<0.0001])$. Multivariate analysis showed pretreatment TLC $>1,000$ cells/mm3 was independent predictors of DFS (hazard ratio $[\mathrm{HR}]=0.39 ; 95 \%$ confidence interval $[\mathrm{CI}]=0.26-0.59 ; \mathrm{p}<0.001)$ and OS (HR=0.59; 95\% CI=0.42-0.84; p=0.006) after adjusted with age, stages of disease and tumor size.

Conclusion: Pretreatment TLC were associated with treatment response, and also the survival outcome in patients with locally advanced stage cervical cancer treated with definite CCRT.

Poster (C08)

Rare Tumors \& Metastatic Tumors

https://doi.org/10.3802/jgo.2021.32.S1.c08

\section{Primary diffuse large B-cell lymphoma of uterine cervix presenting with bulky cervical mass: a case report ana review}

\footnotetext{
A-jaree Senthong, 'Suwapee Watcharahirun

Surin Hospital, Surin, Thailand (ajaree0348@gmail.com)
} 